
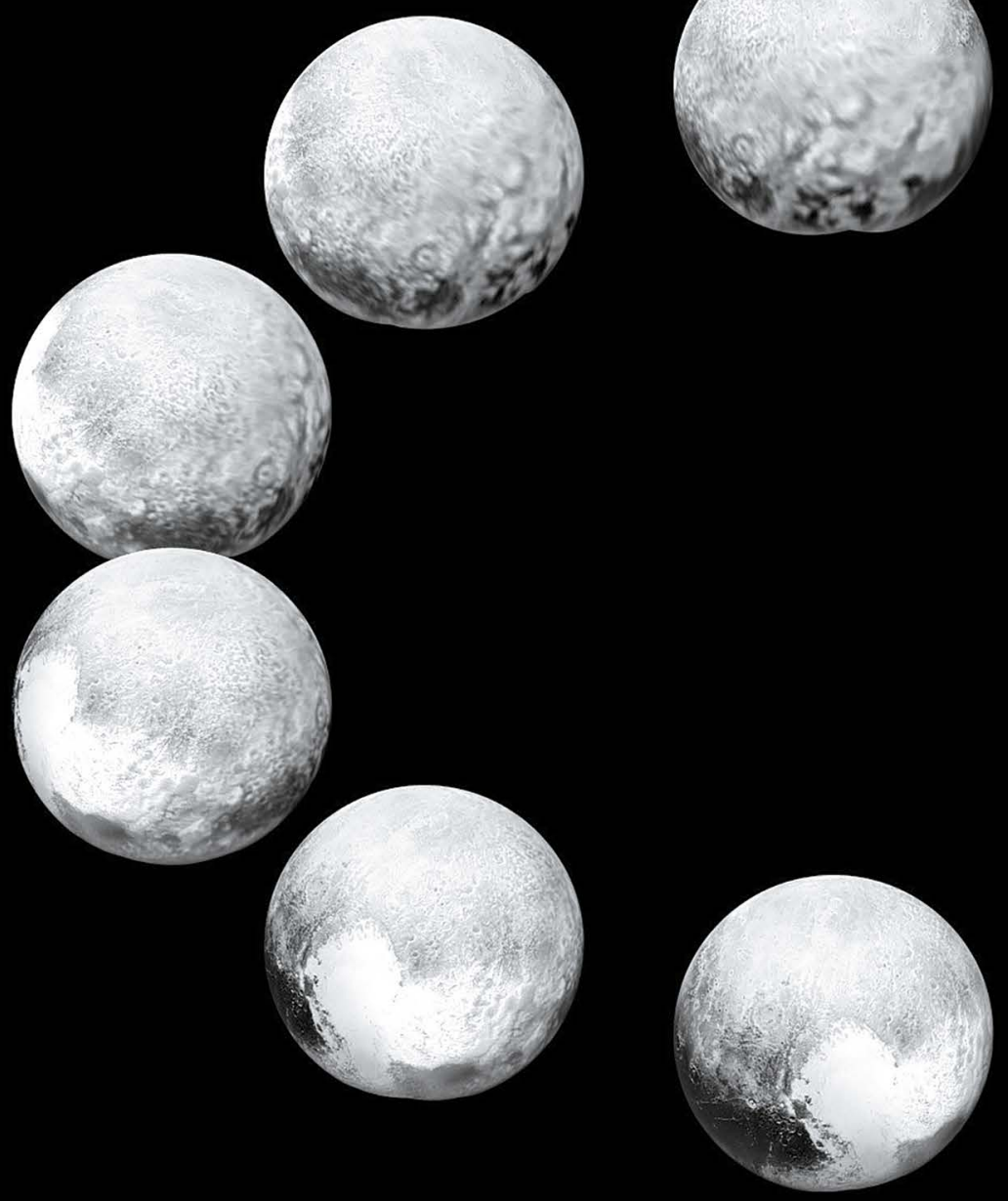

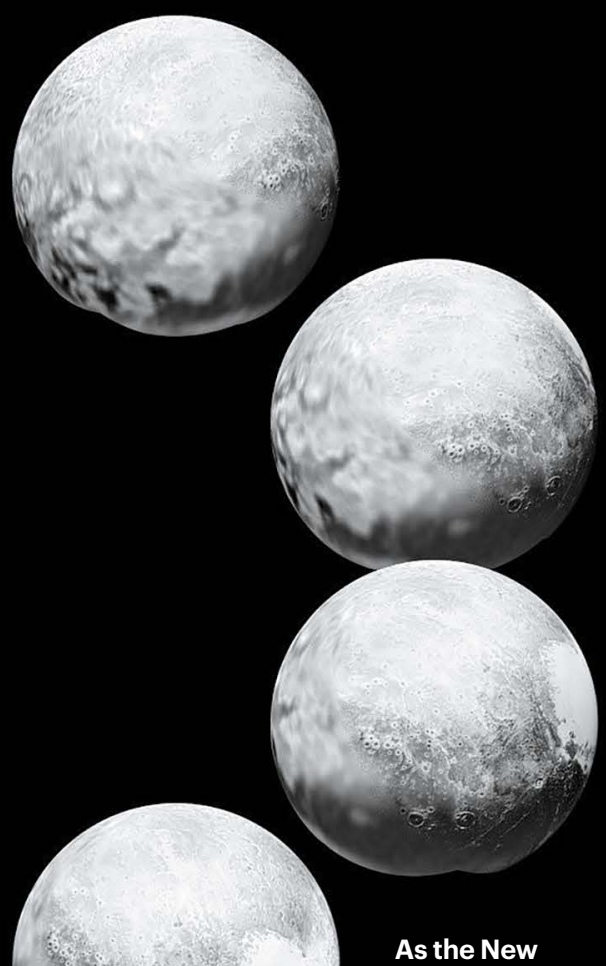

Horizons spacecraft approached Pluto in 2015, it captured one part - the 'near side' - in high resolution (bottom) and the 'far side' at a lower resolution (top).
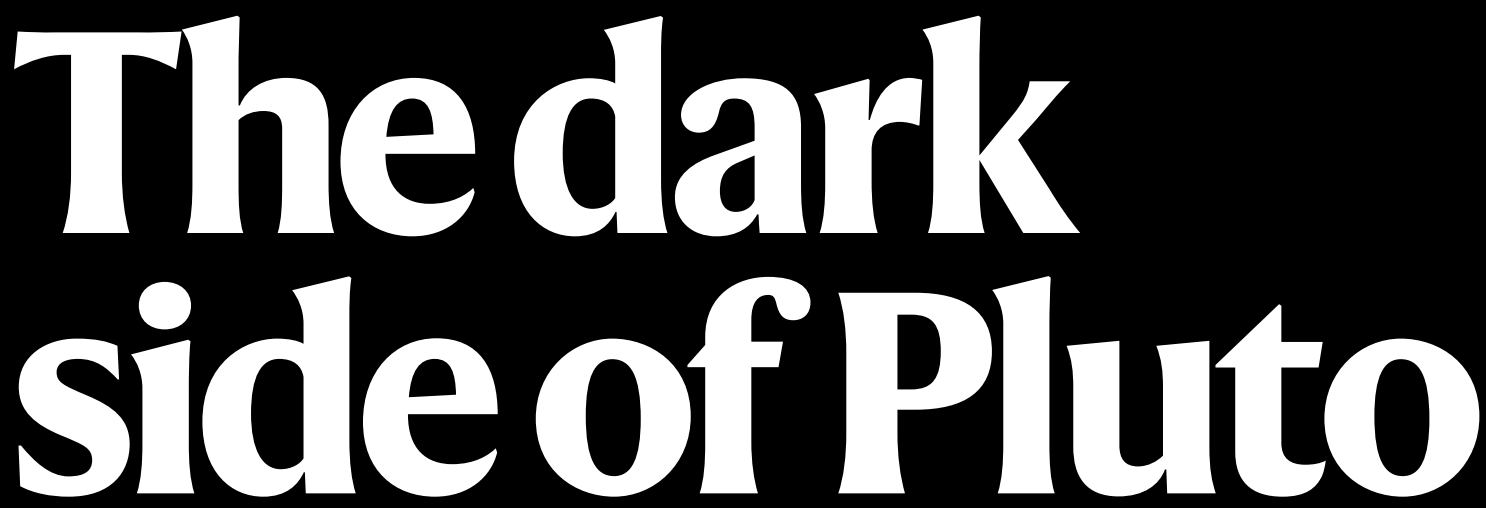

Five years after a jaw-dropping fly-by, images of the dwarf planet's far side reveal possible signs of liquid water, mysterious shards of ice and new theories for the frigid world's birth. By Shannon Hall 


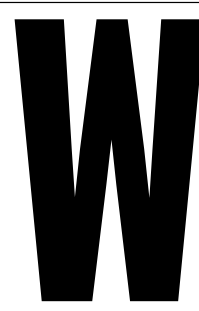

hen NASA's New Horizons spacecraft zipped past Pluto in 2015, it showed a world that was much more dynamic than anyone had imagined. The dwarf planet hosts icy nitrogen cliffs that resemble the rugged coast of Norway, and giant shards of methane ice that soar to the height of skyscrapers. Cracks deeper than the Grand Canyon scar the surface, while icy volcanoes rise taller than Mount Everest. In one part of the distant orb, the spacecraft's cameras captured a giant heart-shaped feature that caused a collective swoon among countless fans on Earth.

"I expected Pluto to be a scientific wonderland, but it did not have to be so beautiful," says Leslie Young, a planetary scientist at the Southwest Research Institute in Boulder, Colorado, and a deputy project scientist on the New Horizons mission.

Although scientists caught that first jaw-dropping glimpse nearly five years ago, they are still seeing images of the world for the first time.

When New Horizons reached the dwarf planet, the craft was moving at 52,000 kilometres per hour, so fast it was able to capture close-ups of only one side of Pluto - the hemisphere that the Sun illuminated at the time. The other was temporarily shrouded in shadow. Now that scientists have scrutinized those 'near-side' close-ups, they are beginning to analyse the other half, which the spacecraft photographed days before it shot past. Researchers call that hemisphere the far side, or even the dark side.

Although the images aren't as clear as those taken later (in which features as small as roughly 75 metres can be seen), they still reveal terrain at resolutions ranging from 2 to 30 kilometres. At best, that's 250 times better than the pictures shot by the Earth-orbiting Hubble Space Telescope. "It's still really juicy data to look at," says Harold Weaver, a planetary scientist at Johns Hopkins University in Baltimore, Maryland, and a New Horizons project scientist. "And it's the best we'll have for another 30-40 years." (That's assuming another spacecraft is sent to Pluto, and soon.)

With hundreds of images to analyse, scientists have a new view ${ }^{1}$ of this active world - one that provides crucial insight into the hazy details of how it formed, whether there's an ocean hiding beneath its icy crust and the complex ways that compounds freeze out of the atmosphere and sculpt its surface. The data even bolster the argument that the chilly world just might be fit for life.

But the images present a set of puzzles. The skyscraper-like shards of ice, for example, were previously seen on the near side, but now appear to circle Pluto. This makes their origin one of the biggest mysteries on the dwarf planet.

"Pluto is the gift that keeps on giving," says Richard Binzel, a planetary scientist at the Massachusetts Institute of Technology in Cambridge and a New Horizons co-investigator.

\section{A splashy start}

When Clyde Tombaugh, a young astronomer at Lowell Observatory in Flagstaff, Arizona, discovered Pluto 90 years ago, it was a speck of light barely visible to the largest groundbased telescopes. It wasn't until 1988 that a chance alignment gave astronomers a lucky break, when Pluto happened to cross in front of a distant star.

As the starlight filtered through Pluto's atmosphere, scientists were able to disentangle the molecules there (including nitrogen, carbon monoxide and methane). Then in 1996, the Hubble Space Telescope helped scientists to finally see surface details at a resolution of 500 kilometres. Although

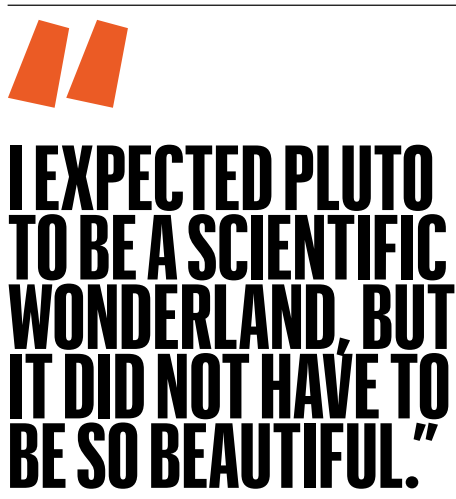

fuzzy, the images revealed a world - at that time still defined as a planet - that had more large-scale contrast than any other in the Solar System, except Earth.

It was a tantalizing hint that suggested Pluto might be a dynamic world - and was quickly verified in July 2015 when New Horizons famously spotted a heart-shaped feature just north of the near side's equator. Within the heart's 'left ventricle' is Sputnik Planitia - an icy basin, churning and flowing with massive glaciers, that scientists now know exerts an extraordinary influence over Pluto's activity. As sunlight warms the frozen plain, a pulse of ice sublimates into vapour that wafts upwards, before dropping back down at the end of the day. The heart might have even knocked Pluto on its side.

Shortly after the first images of the near side arrived at Earth, Francis Nimmo, a planetary scientist at the University of California, Santa Cruz, and his colleagues realized that Sputnik Planitia was in a strange place: it is aligned almost exactly opposite Pluto's largest moon, Charon. It could be an accident, but the likelihood of that is a mere $5 \%$. Instead, models suggest that when the basin formed, an underground ocean began to well up into the chasm. Afterwards, nitrogen gas in Pluto's atmosphere condensed and froze in the frigid basin. The weight of the new water and ice created a heavy load that tipped Pluto into its current alignment ${ }^{2}$.

The idea of a subsurface ocean has existed for some time, but the far-side images have helped to bolster this idea. Some of the strongest evidence comes from a feature

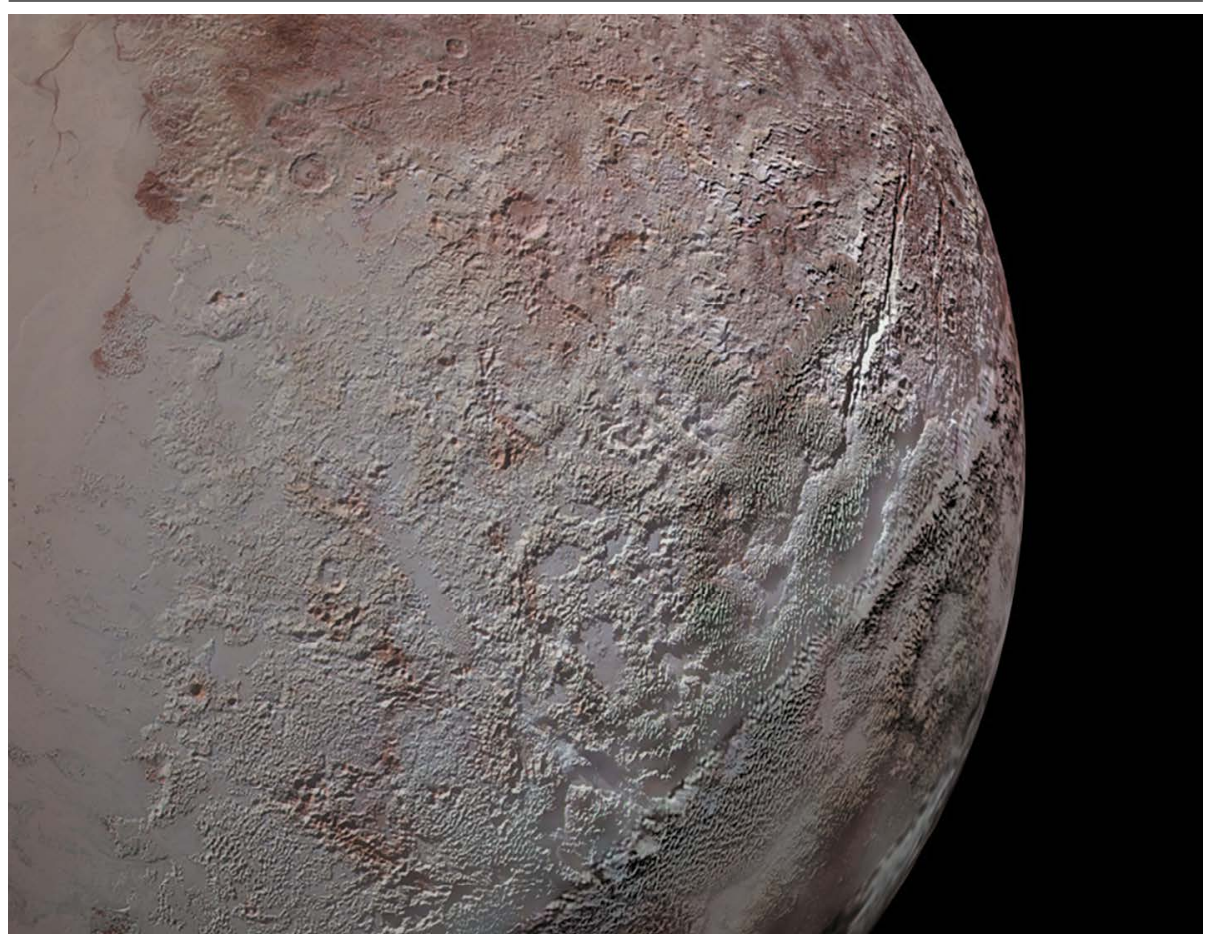

Methane-ice ridges called bladed terrain fill the right part of this image of Pluto's near side. 


\section{Feature}

known as chaotic terrain - a muddled mess of ridges, cracks and plains on the exact opposite side of Pluto from Sputnik Planitia (see 'Coming into focus').

Scientists have seen such pairs before - on Mars, Mercury and Europa, one of Jupiter's moons - where they suspect that a collision from an asteroid or a comet sent seismic waves racing through, and around, the body. Once those quakes converged on the opposite horizon, they tore up the surface in ways resembling what appears on Pluto's far side.

To test the origins of this chaotic terrain, Adeene Denton, a graduate student in planetary geology at Purdue University in West Lafayette, Indiana, simulated how an asteroid impact would send shock waves across the dwarf planet. The work, which was presented virtually at the Lunar and Planetary Science Conference this March, verifies that such a collision would have created the terrain, but with one caveat: it would have been possible only if Pluto had a 150-kilometre-thick subsurface ocean of liquid water.

Oliver White, a planetary scientist at the SETI Institute in Mountain View, California, who built a geologic map of the far side but was not involved in the study, argues that, although the feature does seem to resemble those seen elsewhere in the Solar System, the resolution in the image is low. And other scientists agree. “We will never know until we go

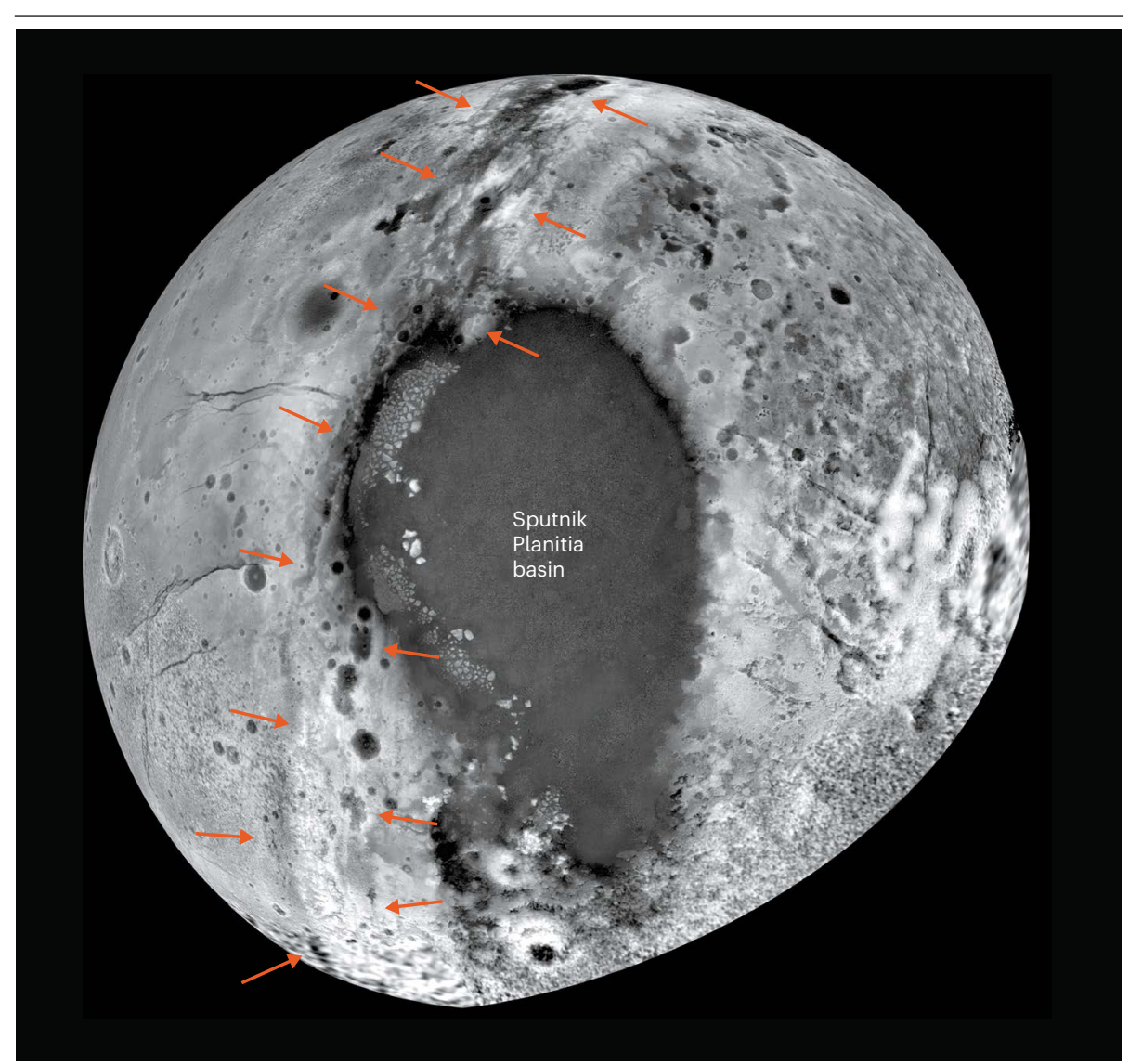

A system of ridges and troughs (orange arrows) appears to wrap around much of Pluto.

back, someday," says William McKinnon, a planetary scientist at Washington University in St. Louis, Missouri, who is deputy lead of the New Horizons geology team.

\section{All cracked up}

Yet Pluto has some geological oddities - in particular, a large number of cracks discovered on the far side - that add support to the existence of a hidden ocean, and even shed light on how it formed.

Scientists who have theorized about Pluto's ocean have long assumed that it had a 'cold start', in that the ocean was frozen when the dwarf planet formed. Later, it would have melted under the heat provided by decaying radioactive elements in its rocky core. In this scenario, the ice would have contracted as it melted - leading to ripples on the surface similar to the wrinkles that appear on an apple left in the refrigerator, Nimmo says. Then, the ice would have expanded as it refroze

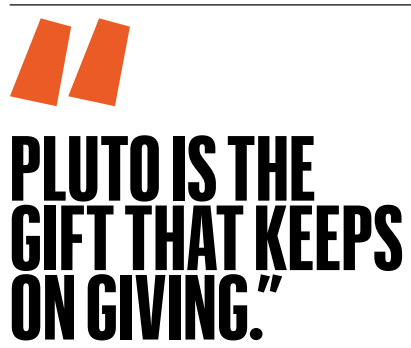

\section{PLUTOISTHE GIFT THAT KEEPS ONGIVING.}

- resulting in cracks on the surface. If this scenario is correct, images of Pluto's surface should reveal older wrinkles and newer cracks. But New Horizons photographed only cracks, suggesting that the dwarf planet's ocean began as a liquid and has partially frozen over time.

In particular, images of the far side reveal a giant crack that stretches up the near side of Pluto. It now seems to run across the north pole and back down towards the south pole on the far side, thus wrapping around the entire dwarf planet ${ }^{1}$. It's similar to the East African Rift System, which is cleaving that continent in two. Unlike the one on Earth, Pluto's rift is not a sign of shifting continents, but is probably a scar from the freezing and ever-expanding ocean. Because the crack is extremely old, it is clear that the liquid ocean began to chill immediately, Nimmo says.

If true, the ocean might even be ripe for the existence of life. Observations of water that had probably gushed out of the ocean on the near side show that it is red - hinting that it's stained with organic molecules. Although that might seem impossible on a world such as Pluto, laboratory experiments have shown that radiation similar to solar wind or cosmic rays can create complex organic matter that is reddish brown ${ }^{3}$. And, if ammonia is present, it is possible to form molecules that are crucial for life, including the bases that are present in RNA and DNA.

So when Dale Cruikshank, a planetary scientist at the NASA Ames Research Center in Moffett Field, California, and deputy lead of the New Horizons composition team, and his colleagues revealed in the journal Astrobiology last year that Pluto's near-side ices were both red and laced with ammonia ${ }^{4}$, it was a crucial sign that the orb might be replete with organic molecules. The theory, which has gained a lot of traction among planetary scientists, doesn't mean that life got a start on Pluto, Cruikshank says, but that if it were introduced to Pluto, it could survive. "Before New Horizons got to Pluto, I would have never in a million years expected that there would be an article about Pluto in Astrobiology," says Cathy Olkin, a planetary scientist at the Southwest Research Institute and a deputy project scientist on the mission.

Results from the far side bolster this theory. They reveal a red band, probably of organic material, that stretches across the equator - corresponding to an area with the most sunlight and the most temperate climate ${ }^{1}$. "So the astrobiologists say you need liquid water, organics and a source of energy" to support life, says Alan Stern, a planetary scientist at the Southwest Research Institute and the principal investigator of the mission. “There's a pretty good case for liquid water, and it's getting stronger. Now we have a case for organics. So that's two of the three 


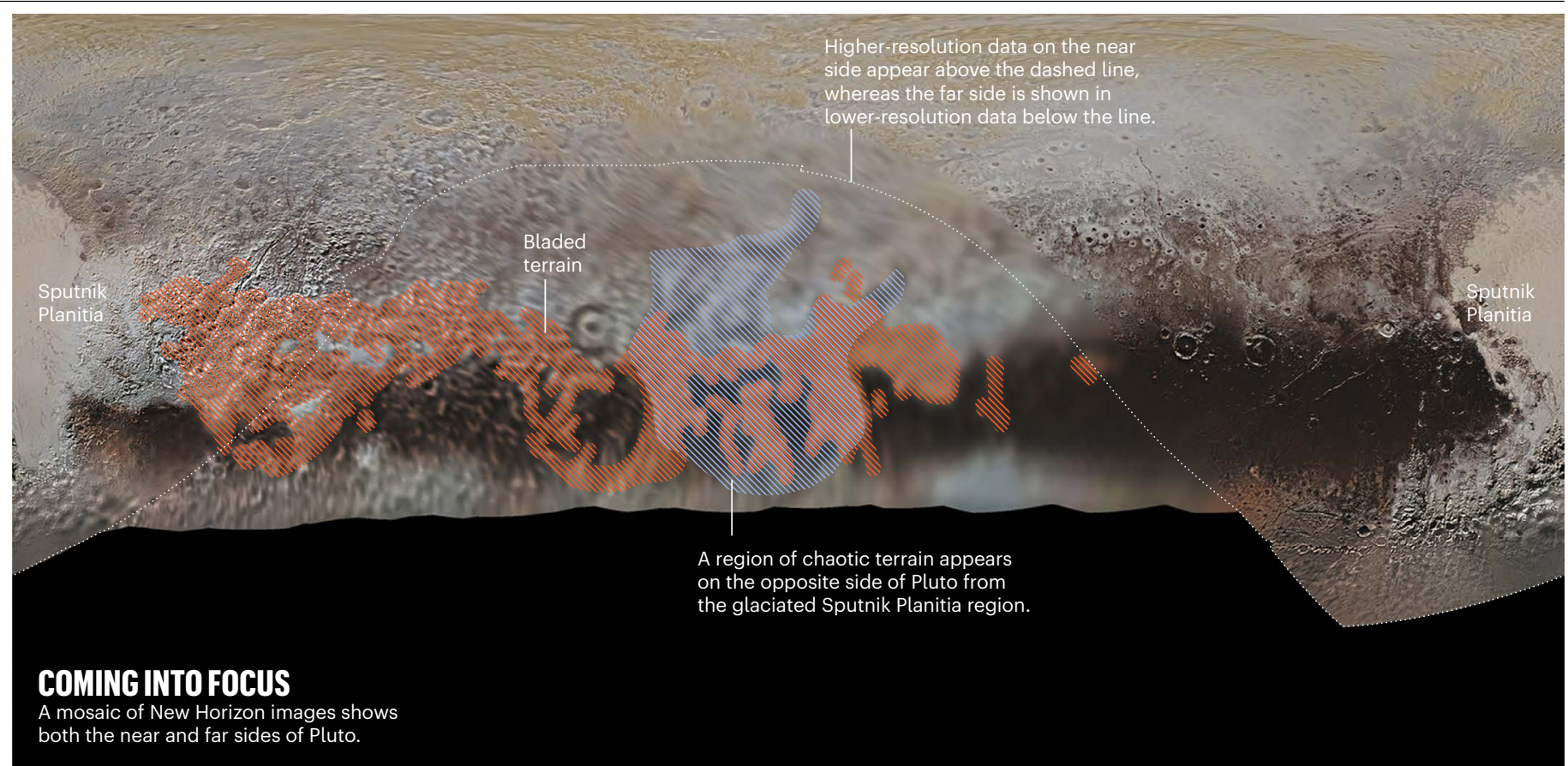

boxes checked, which is a very important step forward."

\section{Pluto's sharp secret}

Beyond helping to explore Pluto's potential for life, the far-side measurements have generated a number of mysteries.

When the images from New Horizons first reached Earth, scientists noticed a bizarre terrain that consisted of skyscraper-sized shards of ice ${ }^{1}$ along the near side's easternmost region. These evenly spaced ridges are only a few kilometres apart, yet rise sharp and knife-like into the sky, occasionally soaring as high as one kilometre - roughly three times the height of the Empire State Building in New York City. They can be as long as 30 kilometres. "This would be a nightmarish landscape to try and navigate," White says.

But they were nothing more than a blip on the map until scientists caught a glimpse of the far side. Although the latest map is blurry (with a resolution too poor to actually see the individual ridges themselves), it's clear that the bladed terrain wraps all the way around the far side and pops out again on the western edge of the near side in a region that was previously overlooked ${ }^{1}$. On the far side, they cover an area that is 3.5 times larger than their extent on the near side - making them one of the biggest mysteries on Pluto.

"When we discovered that this is such a widespread terrain type, it became much more important to understand it instead of being kind of an interesting exotic footnote," Stern says.

Spectral data reveal that the blades are composed of methane ice and create a belt around the equator - at least on plateaus and mountains. But how they form remains a mystery. It might be that methane froze out of the atmosphere in the same way that frost freezes on the ground on Earth. Or perhaps they're vestiges of an old layer of methane ice that was eroded by the glaring sunlight.

Some researchers have focused on the latter explanation, because the sharp ridges resemble structures that form in the high Andes albeit on a radically smaller scale. The South American versions, called penitentes, are only

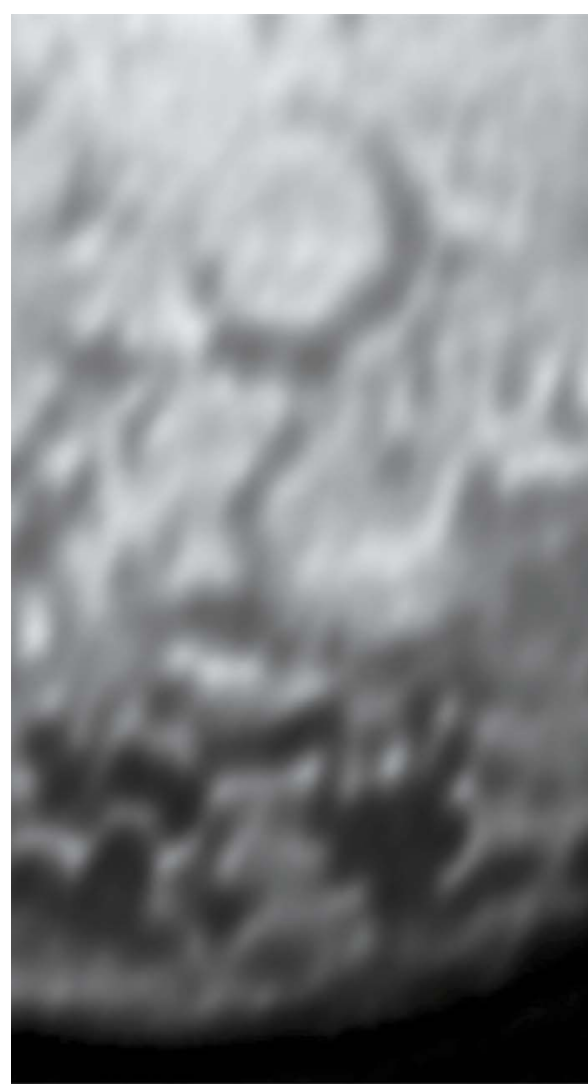

A close-up of Pluto's chaotic terrain on its far side. a few metres tall and form in high-altitude regions. Because they angle towards the Sun and develop along the Equator, where solar radiation is strongest, scientists suspect that light has a hand in the penitentes' formation. Sunlight is thought to drive sublimation essentially carving out valleys between bladelike ridges.

A similar theory for the features on Pluto was put forth in 2017 by Jeffrey Moore, a planetary scientist at the NASA Ames Research Center and a New Horizons co-investigator, and his colleagues ${ }^{5}$. But co-author Tanguy Bertrand, also at the NASA Ames Research Center, argues that it is hard to use that process to explain all of the blades' characteristics. Although some are oriented in the expected north-south direction - consistent with the Sun's path across Pluto's sky - others are oriented northeast-southwest or east-west. That could argue against erosion by sunlight. But the other possibility, precipitating methane, also has problems, Bertrand argues, because there is no bright frost.

What is clear is that the icy shards (and Pluto's landscape in general) cannot be understood without closely studying the dwarf planet's weather. Just last year, Bertrand and his colleagues published a climate model ${ }^{6}$ showing that methane accumulates at higher altitudes, whereas nitrogen builds up in the low atmosphere - explaining why the Sputnik Planitia basin is rich in nitrogen ice, but the bladed terrain is dominated by methane ice.

That's different from Earth, where liquid water fills the low-lying oceans and water ice coats the highest mountain tops. On our planet, wind can travel upslope - thus transporting water from low to high altitude. But on Pluto, such a manoeuvre cannot happen. Because its atmosphere is warmer 


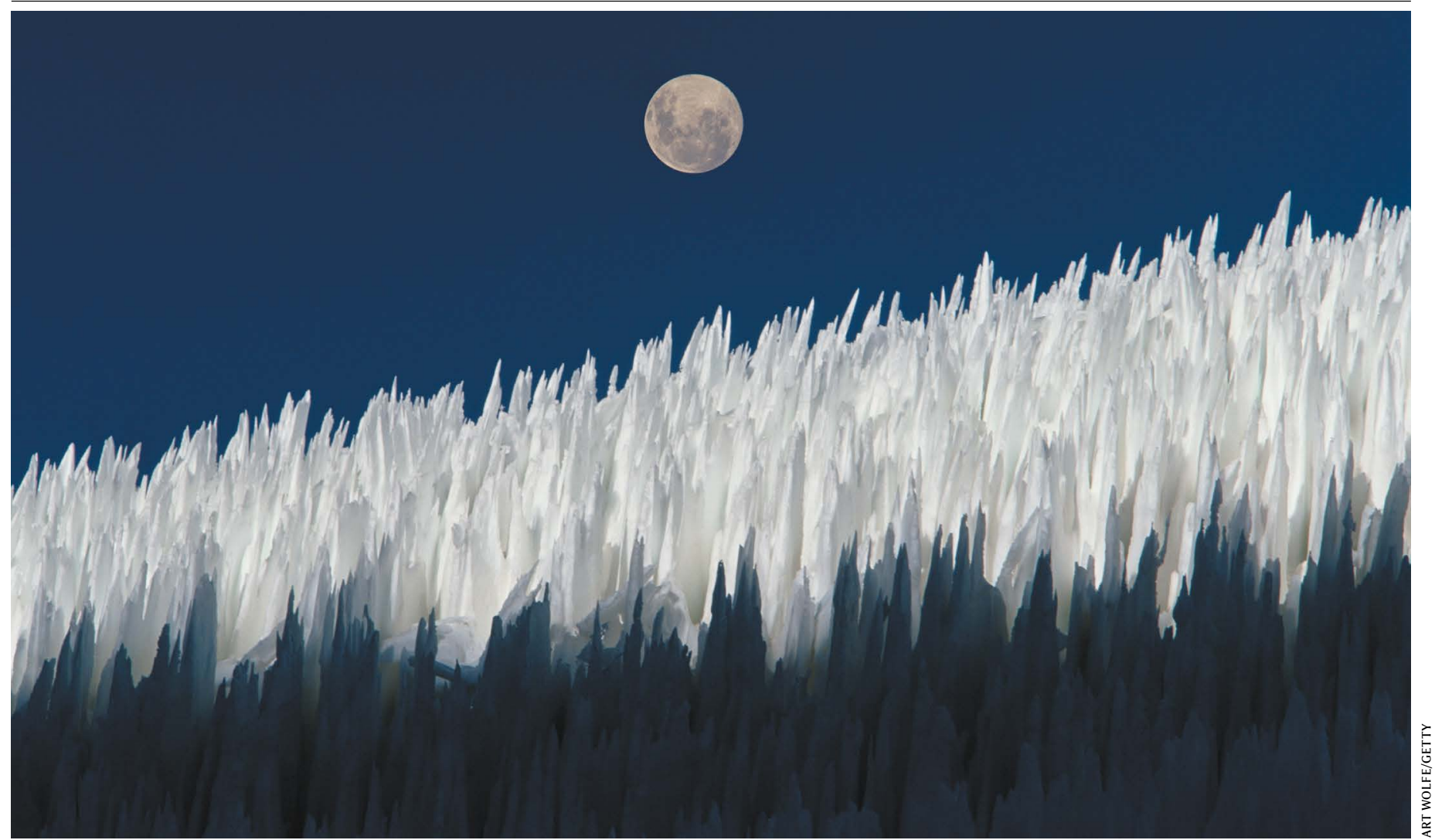

Icy peaks called penitentes in the Andes are just a few metres tall, but might be analogues of much larger ridges in Pluto's bladed terrain.

than the surface, downslope winds dominate - meaning that the low-lying nitrogen could never travel up the highest peaks, and high-flying methane would condense on the tallest mountains before it reached the lower parts of the surface.

Moreover, the bladed ridges seem to be ancient features that have grown over long stretches of time. Not only would it take tens of millions of years for ice to either condense or sublime into such tall features, but their location spans a wide range of latitudes. Changes in the past climate might explain how the bladed terrain's location moved and even formed but there are plenty of details that need to be teased out.

One of those is Pluto's wacky seasons. On Earth, the seasonal cycle is controlled by changes in the amount of direct sunlight as one hemisphere of our planet tilts towards or away from the Sun - not by the planet's orbital path. On Pluto, the seasons are controlled by its tilt, and by changes in its elliptical orbit as the dwarf planet swings towards the frigid edges of the outer Solar System once per year and then zooms back in towards the Sun (nearly cutting its distance from the Sun in half). Because both of those parameters change over time, Pluto will nestle close to the Sun at the same time that a particular hemisphere is pointed towards the Sun once every 900,000 years - two effects that conspire to create a 'super season' in that hemisphere. Bertrand suspects that this effect could have perturbed

\section{"THISWOULDBE (1) LANDSCAPETO TRYAND NAVIGATE.}

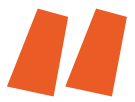

the latitude where the bladed terrains formed, but scientists cannot say for sure because the calculation of orbital parameters is difficult to estimate beyond the past ten million years.

It's an open question that could inspire future work. Now that the dwarf planet has been further mapped, a handful of scientists are trying to tease out details from its past. Binzel is scouring the near-side images of Pluto to better understand its current seasons. By comparing its different regions, he hopes to predict any changes we might expect should we send an orbiter to Pluto in another 50 years. "It's like trying to do a Pluto forecast," he says. "I'm not sure whether I'll get to see that myself, but it's nice to leave Easter eggs of things to look for the next time we get back to Pluto."

Binzel is certain that we will eventually launch another probe to the dwarf planet. Indeed, many argue that it's almost inevitable given the numbers of questions that New Horizons left in its wake. To that end, NASA recently awarded scientists the opportunity to study the feasibility of an orbiter, which would allow them to map all of Pluto in detail and even to watch it change over time. This study is a long way from being an actual mission, warns Carly Howett, a planetary scientist at the Southwest Research Institute, who is leading the effort. It probably wouldn't launch until the 2030s, or even 2040s, if NASA decides to pursue the mission. Then, it would be a 15 -year cruise to the dwarf planet.

In the meantime, scientists will continue to build better climate models to match the data they have. They will work in the lab to try to simulate the chemistry in Pluto's atmosphere and potential ocean. And they will swivel the James Webb Space Telescope, NASA's successor to Hubble, towards the dwarf planet after the satellite launches next year. Although Webb will not be able to image Pluto at a very high resolution, it will be able to use longer wavelengths and is thus likely to reveal something new.

"I think there's just mouth-watering science to be done," Stern says.

Shannon Hall is a freelance science journalist in Colorado.

\footnotetext{
. Stern, S. A. et al. Preprint at https://arxiv.org/ abs/1910.08833 (2020).

2. Nimmo, F. et al. Nature 540, 94-96 (2016).

3. Miller, S. L. Science 117, 528-529 (1953).

4. Cruikshank, D. P. et al. Astrobiology 19, 831-848 (2019).

5. Moore, J. M. et al. Icarus 300, 129-144 (2018).

6. Bertrand, T. et al. Icarus 329, 148-165 (2019).
} 